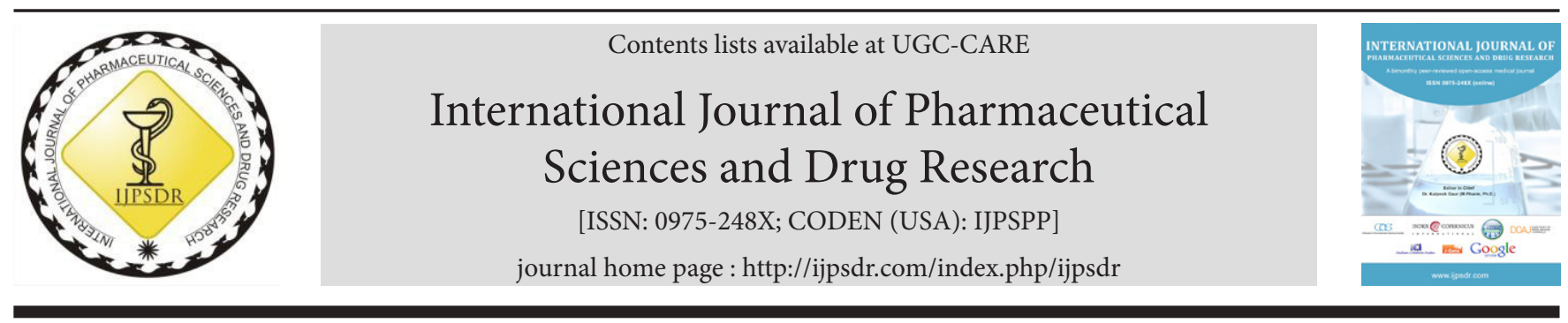

Research Article

\title{
Development and Evaluation of Novel Bionanoparticles loaded with Nanosized Lamotrigine by using the Novel Isolated Biopolymer from Phaseolus vulgaris Seeds
}

\author{
Sushant Kumar ${ }^{1}$, Nookala Venkala Satheesh Madhav ${ }^{2}$, Anurag Verma ${ }^{3}$, Kamla Pathak ${ }^{1}$ \\ ${ }^{1}$ Faculty of Pharmacy, Uttar Pradesh University of Medical Sciences, Saifai, Etawah-206130, Uttar Pradesh, India \\ ${ }^{2}$ Faculty of Pharmacy, DIT University, Dehradun-248009, Uttarakhand, India \\ ${ }^{3}$ School of Pharmaceutical Sciences, IFTM University, Moradabad-244001, Uttar Pradesh, India
}

\begin{tabular}{l} 
A R T I C L E I N F O \\
\hline Article history: \\
Received: 14 February, 2020 \\
Revised: 11 April, 2020 \\
Accepted: 30 April, 2020 \\
Published: 30 May, 2020 \\
Keywords: \\
Bionanosuspension, \\
Biopolymer, \\
Lamotrigine, \\
Phaseolus vulgaris. \\
D0I: \\
10.25004/IJPSDR.2020.120306
\end{tabular}

\section{INTRODUCTION}

The word biopolymer has drawn the attention of researchers as a novel biomaterial for development and designing of targeted drug delivery systems. ${ }^{[1]}$ Nowadays, the drawbacks associated with synthetic and semisynthetic polymers are very challenging problems in drug delivery system designing. The synthetic and semisynthetic polymers are the first choice in development of targeted drug delivery systems. But these polymers have a number of side effects which may produce different patient incompatibility.

\begin{abstract}
A B S T R A C T
The aim of this research was to isolate and characterize the novel biopolymer from Phaseolus vulgaris seeds and to formulate lamotrigine loaded bionanoparticles using this biomaterial. The biopolymer was isolated by simplified and economical process and analyzed for different physicochemical and spectral properties. The nanosizing of lamotrigine was done by bath sonication method, and its particle size in nano-range was screened by UV method. The bionanosuspension loaded with nanosized lamotrigine was prepared by bath sonication for 15 cycles ( 1 cycle equal to 3 minutes). The formulated bionanosuspension loaded with lamotrigine using biopolymer was compared with lamotrigine loaded bionanosuspension by using hydroxypropyl methyl cellulose (HPMC). The bionanosuspension was subjected for $\mathrm{pH}$ study, dispersibility study, \% entrapment efficiency, nanosizing screening, particle size study, and in vitro study for drug release and stability study of formulated bionanosuspension. The best formulation LPVu7 showed up to $90.36 \pm 0.9 \%$ drug release in 36 hours having t $50 \%$ of 17 hours and $t 80 \%$ of 29 hours with $\mathrm{r}^{2}$ value of 0.9927 as compared to release from LSP10 with $92.43 \pm 0.68 \%$, having t $50 \%$ of 16 hours and t $80 \%$ of 28 hours with $\mathrm{r}^{2}$ value of 0.9951 in 36 hours. The results reveal that isolated biopolymer may be used as an alternative to synthetic polymer because of its novel inbuilt bioretardant cum biostabilizing properties. The formulated bionanosuspension is feasible for delivering the nanosized lamotrigine in sustained manner for prolonged time for long term treatment of epilepsy.
\end{abstract}

\footnotetext{
${ }^{*}$ Corresponding Author: Mr. Sushant Kumar

Address: Faculty of Pharmacy, Uttar Pradesh University of Medical Sciences, Saifai, Etawah-206130, Uttar Pradesh, India

Email $₫:$ k.sushant25@gmail.com

Relevant conflicts of interest/financial disclosures: The authors declare that the research was conducted in the absence of any commercial or financial relationships that could be construed as a potential conflict of interest.

Copyright (C) 2020 Sushant Kumar et al. This is an open access article distributed under the terms of the Creative Commons Attribution- NonCommercialShareAlike 4.0 International License which allows others to remix, tweak, and build upon the work non-commercially, as long as the author is credited and the new creations are licensed under the identical terms.
} 
degradation products. The use of polycaprolactone with polysorbate 80 produced the granulomatous foreign body reaction in liver and spleen in rats. Polycaprolactone shows the slow degradation rate, poor mechanical properties, and low cell adhesion.

So the biopolymers isolated from different natural sources, like flowers, seeds, barks, and rhizomes have drawn the attention of researchers in designing novel drug delivery systems. ${ }^{[2]}$

The isolated biopolymer has novelistic polymeric properties; it may be used as an intelligent biomaterial for development of drug delivery systems. Since these biopolymers are isolated from natural sources, these are biodegradable and biocompatible in nature. ${ }^{[3]}$ The researches have proven the other novel properties, like good biodegradability, mucoadhesivity, filmability, retardibility, and release rate controlling properties. ${ }^{[4]}$ Since these have excellent release rate controlling properties, these isolated biopolymers may be used for the designing of sustained, controlled, and extended-release drug delivery systems. ${ }^{[5,6]}$ Biopolymer from the natural source, like $P$. vulgaris may be used for the formulation of bionanosuspension for the delivery of lamotrigine. ${ }^{[7,8]}$

The nanoparticles (NPs) have attracted the researchers as a smart tool for drug targeting to brain in an efficient way. NPs having the particle size in nano range may cross the BBB in efficient way. So it can be used as a novel tool for targeting the antiepileptic drug-like lamotrigine for ear to brain administration. Lamotrigine is available as tablets ${ }^{[9]}$ and research reports reveal delivery of drug from the formulated microcapsules and nanoparticles by using the synthetic and semi-synthetic polymers. ${ }^{[10]}$

Epilepsy is a neurological disorder in which the brain condition becomes abnormal and shows repeated, uncontrolled, and sudden changes in brain. In epilepsy, there are abnormal changes in electrical activity of brain. The brain is the vital part of body which controls body activities. The brain is protected by the blood-brain barrier, which controls the movement of various ions and macromolecules inside the brain. ${ }^{[11]}$

In this research work, bionanosuspension having the bionanoparticles loaded with nanosized lamotrigine were aimed at using novel biomaterial from seeds of P. vulgaris. ${ }^{[12,13]}$ The biopolymer isolated from the seeds of $P$. vulgaris. The isolated biopolymers was characterized, formulated as bionanosuspension and assessed for its potential for delivery of lamotrigine for its potential for efficient therapeutics of epilepsy.

\section{Materials AND METhods}

\section{Materials}

Lamotrigine was a gift sample from Affy Pharma Pvt. Ltd., Baddi. The P. vulgaris seeds (rajma seeds) were procured from the local market, Lucknow, Uttar Pradesh. The remaining solvents and chemicals used were of analytical reagent grade.

\section{Isolation of $P$. vulgaris Biopolymer}

Two hundred grams of seed was weighed and soaked in double-distilled water overnight. The swollen seeds were taken, and their outer covering was removed. The uncovered seeds were grounded in a grinder as a paste. If necessary, small quantity of distilled water may be added during the grinding. This paste was filtered through the muslin cloth. The collected filtrate was centrifuged at 5,000 rpm for 10 minutes. After centrifugation, the supernatant was taken. Centrifugation was done to remove any residue. Then, half of the supernatant was treated with acetone in 1:1,1:2, and 1:3 ratios. Another half of supernatant was treated with the methanol in the same ratios as acetone. Then these were placed in refrigerator overnight. The mixtures were centrifuged at 5,000 rpm for 30 minutes. The biomaterial as sediment was collected by discarding the supernatant liquid and air-dried. The product was stored in desiccators for 48 hours. The obtained biomaterial was passed through sieve number 200 and stored for further use. This procedure was optimized by repeating it six times, and then percentage yield was calculated.

\section{Characterization of Isolated Biopolymer}

The physicochemical properties of isolated biopolymer were characterized for color, odor, taste, and solubility. ${ }^{[14]}$ The chemical tests for presence of carbohydrate, starch, and proteins were also performed. The isolated biopolymer was also characterized for scanning electron microscopy (SEM) analysis, differential scanning calorimetry (DSC) testing, infrared spectroscopy (IR) spectroscopy, mass spectroscopy, and nuclear magnetic resonance spectroscopy (NMR).

\section{Scanning Electron Microscopy (SEM) Analysis}

The isolated biopolymer was analyzed by scanning electron microscope. In SEM analysis, the external surface and internal structure was characterized. The small quantity of biopolymer was taken and fixed on aluminum studs, and the coated with gold with the help of coater sputter under vacuum. The scanning electron micrographs were taken for the biopolymer under observation.

\section{Fourier Transform Infrared Spectroscopy (FTIR) Spectroscopy of Isolated Biopolymer}

The FTIR spectroscopy was done by preparing the $\mathrm{KBr}$ discs. $1 \mathrm{mg}$ of isolated biopolymer was taken and mixed with $100 \mathrm{mg}$ of dried and desiccated solid KBr. The mixture was mixed in mortar and pestle and placed in IR lamp to remove any moisture. The mixture was converted into disc under the pressure of 10 tons. The prepared disc was placed in a disc holder in the path of IR radiation. The spectrum was recorded in the range of 4,000 to $200 \mathrm{~cm}^{-1}$.

\section{Differential Scanning Calorimetry (DSC) Testing}

The DSC testing is the thermal analysis technique, in which the heat flow into or out of the sample is determined as the function of temperature. Here, the sample was taken 
and exposed to controlled temperature program. The glass transition temperature was determined. The heat flow range was 50 to $300^{\circ} \mathrm{C}$. The DSC thermogram was recorded.

\section{NMR Spectral Analysis}

The NMR spectroscopy was done for spectral analysis of isolated biopolymer. The sample was dissolved in specific solvent, like $\mathrm{CDCl}_{3}$. The mixture was pumped in the instrument at high rate flow. The valve switch was used to stop the flow. The measurement was performed. After the finishing of measurement, the spectrum was processed and analyzed in an automation computer.

\section{Nanosizing of Lamotrigine}

$500 \mathrm{mg}$ of lamotrigine was taken and dissolved in $25 \mathrm{~mL}$ of methanol. The clear solution was bath sonicated for optimized sonication cycle for 15 cycles (one cycle was for three minutes) continuously. The bath sonication was done at $250 \mathrm{~Hz}$ frequency at room temperature. During sonication, $25 \mathrm{~mL}$ of purified water as added slowly drop by drop till precipitation was obtained. The obtained precipitate was subjected for centrifugation for 15 minutes at 4,000 rpm for complete recovery of nanosized drug. ${ }^{[15,16]}$ During the optimization process at each cycle, $\%$ transmittance was noted in order to know the minimum cycles required for nanosizing of the drug. Later, the mixture was subjected for centrifugation in order to recover the nanosized lamotrigine. The recovered nanoparticles were observed for physical appearance, permeability through egg membrane. Further, the nanoparticle was stored in airtight container for further use.

The physical appearance of nanosized lamotrigine was observed, and permeability study was conducted.

\section{Permeability through the Egg Membrane}

The $5 \mathrm{mg} / \mathrm{mL}$ of nanosized lamotrigine solution was prepared in phosphate buffer, $\mathrm{pH} 7.4$, and added ( $4 \mathrm{~mL}$ ) in the donor compartment, and tied the donor compartment with the egg membrane. Phosphate buffer, $\mathrm{pH} 7.4$ solution, and added to the receptor compartment, (Fig. 1). The donor with drug solution was fitted in receptor compartment for the permeation of drug. At specific time interval, the receptor compartment sample was completely replaced with the fresh $100 \mathrm{~mL}$ of the buffer solution. The sampling was done at 1, 2, 4, 6, 8, 10, 24, 36, and 48 hours, and assayed. A graph was plotted between the time and concentration of drug permeated at specific time interval. This procedure was done in triplicate.

\section{Drug-Excipient Interaction}

The drug-biopolymer interaction study was performed by the UV spectroscopy method. The lamotrigine-biopolymer mixture was prepared in ratio of $1: 1,1: 3$, and $3: 1$, and examined by wet and dry method. After mixing, the drug and polymer mixtures were stored at $50^{\circ} \mathrm{C}$ for one hour. In wet method, the mixture was wetted with distilled water $(1 \mathrm{~mL})$ and then dried in oven at $50^{\circ} \mathrm{C}$ for one hour. The dried mixture was treated with methanol in order to dissolve the lamotrigine, and further $\lambda_{\text {max }}$ was determined and repeated for three times. In dry method, the three different ratio of drug-biopolymer in same as wet method was prepared, and then, treated with methanol in order to dissolve the lamotrigine and further $\lambda_{\max }$ was determined. This was repeated for three times. $\lambda_{\max }$ was compared before and after the test for any change.

\section{Formulation of Lamotrigine-loaded Bionanosuspension}

The formulations of bionanosuspension were prepared by using different drug-biopolymer ratio and drugstandard polymer ratio, as given in Tables 1 and 2 . The bionanosuspension was prepared by sonication of

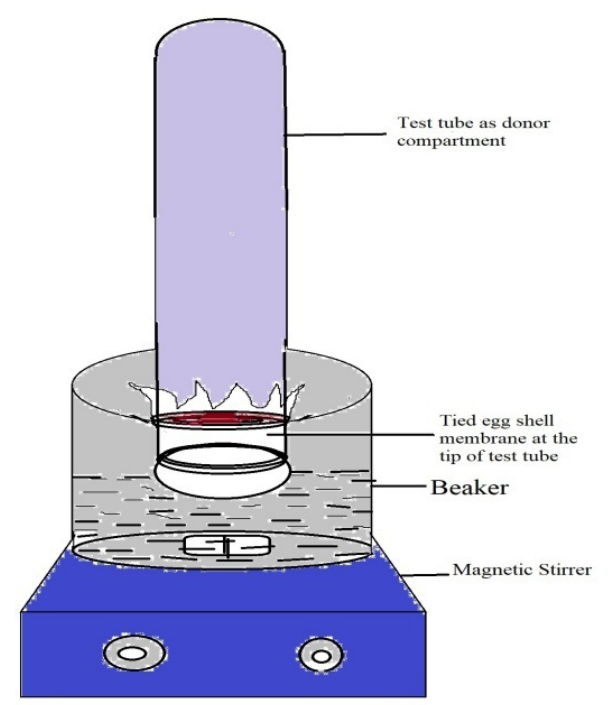

Fig. 1: Assembly for permeation study across egg membrane

Table 1: Formulation table of lamotrigine loaded bionanosuspension using $P$. vulgaris biopolymer

\begin{tabular}{lllllllllll}
\hline Formulation & LPVu1 & LPVu2 & LPVu3 & LPVu4 & LPVu5 & LPVu6 & LPVu7 & LPVu8 & LPVu9 & LPVu10 \\
\hline Drug:biopolymer ratio & $1: 0.8$ & $1: 1$ & $1: 2$ & $1: 3$ & $1: 4$ & $1: 5$ & $1: 8$ & $1: 10$ & $1: 12$ & $1: 15$ \\
Lamotrigine (mg) & 10 & 10 & 10 & 10 & 10 & 10 & 10 & 10 & 10 & 10 \\
Biopolymer (mg) & 8 & 10 & 20 & 30 & 40 & 50 & 80 & 100 & 120 & 150 \\
Polyvinyl alcohol (mL) & 0.5 & 0.5 & 0.5 & 0.5 & 0.5 & 0.5 & 0.5 & 0.5 & 0.5 & 0.5 \\
Sodium benzoate (\%) & 0.5 & 0.5 & 0.5 & 0.5 & 0.5 & 0.5 & 0.5 & 0.5 & 0.5 & 0.5 \\
Double distilled water (mL) & 10 & 10 & 10 & 10 & 10 & 10 & 10 & 10 & 10 & 10 \\
\hline
\end{tabular}


Sushant Kumar et al.

Table 2: Formulation table of lamotrigine loaded nanosuspension using HPMC polymer

\begin{tabular}{|c|c|c|c|c|c|c|c|c|c|c|}
\hline Formulation & $L S P 1$ & LSP2 & LSP3 & LSP4 & LSP5 & LSP6 & LSP7 & LSP8 & $L S P 9$ & LSP10 \\
\hline Drug:polymer ratio & $1: 0.8$ & $1: 1$ & $1: 2$ & $1: 3$ & $1: 4$ & $1: 5$ & $1: 8$ & $1: 10$ & $1: 12$ & $1: 15$ \\
\hline Lamotrigine (mg) & 10 & 10 & 10 & 10 & 10 & 10 & 10 & 10 & 10 & 10 \\
\hline Hydroxypropyl methyl cellulose (mg) & 8 & 10 & 20 & 30 & 40 & 50 & 80 & 100 & 120 & 150 \\
\hline Polyvinyl alcohol (mL) & 0.5 & 0.5 & 0.5 & 0.5 & 0.5 & 0.5 & 0.5 & 0.5 & 0.5 & 0.5 \\
\hline Sodium benzoate $(\%)$ & 0.5 & 0.5 & 0.5 & 0.5 & 0.5 & 0.5 & 0.5 & 0.5 & 0.5 & 0.5 \\
\hline Double distilled water $(\mathrm{mL})$ & 10 & 10 & 10 & 10 & 10 & 10 & 10 & 10 & 10 & 10 \\
\hline
\end{tabular}

the mixture of drug and biopolymer along with other excipients, like polyvinyl alcohol as suspending agent, sodium benzoate as the preservative, purified water, and dextrose as nanosizent. The lamotrigine, P. vulgaris biopolymer, and other excipients were accurately weighed and triturated with addition of the double-distilled water. This mixture was sonicated for three cycles. Then $0.5 \mathrm{~mL}$ of $0.5 \%$ polyvinyl alcohol was added during sonication. The volume was made up to $10 \mathrm{~mL}$ with double distilled water having sodium benzoate 0.1 to $0.5 \%$. Add dextrose if necessary as nanosizing agent and allowed for sonication for 15 cycles. After sonication, the bionanosuspension was refrigerated for two days. If no settlement is there, then it means the formulation is optimized. If settlement is there, then $0.5 \mathrm{~mL}$ of $0.5 \%$ polyvinyl alcohol was again added and allowed for sonication for 10 cycles and refrigerated for 48 hours. The different formulations were prepared, and after optimization according to stability the formulations LPVu1 to LPVu10 were prepared. In the same way, the nanosuspension was also prepared by using lamotrigine and standard polymer, like hydroxypropyl methylcellulose. The nanosuspensions LSP1 to LSP10 were prepared. After formulation, their stability was tested, and then, evaluated for different parameters, including release study.

Standard lamotrigine-nanosuspension was prepared using HPMC polymer, and then, the same was compared with formulated lamotrigine bionanosuspension using biopolymer. Formulated HPMC nanoformulation was subjected for in vitro drug diffusion performance study

\section{Characterization of Lamotrigine-loaded Bionanosuspension}

\section{Dispersibility}

$20 \mathrm{mg}$ of the formulated nanoparticles was taken and dispersed in $20 \mathrm{~mL}$ of the distilled water in a test tube. The time for settling of the dispersed nanoparticles in the bottom was noted, and then again the nanoparticles was redispersed and noticed for the redispersion. After shaking any lump or aggregates or any precipitation, formation was observed. This procedure was done in triplicate.

\section{$p H$}

The $\mathrm{pH}$ of formulated bionanosuspension was evaluated with digital $\mathrm{pH}$ meter (Systronics). ${ }^{[17]}$ The study was done in triplicate, and the mean was taken and checked that the
$\mathrm{pH}$ of the nanosuspension is in the required range or not. This procedure was done in triplicate.

\section{Entrapment Efficacy}

The freshly formulated bionanosuspension was taken and centrifuged at 20,000 rpm in ultracentrifuge. After centrifugation, the supernatant was taken and diluted up to $10 \mu \mathrm{g} / \mathrm{mL}$ appropriately and unincorporated amount of lamotrigine was determined by measuring the absorbance under UV spectroscopy at $307 \mathrm{~nm}$ after appropriate dilution. The amount of drug entrapped in bionanoparticles was determined. For this, amount of free drug in supernatant was subtracted from the initial amount of lamotrigine taken in formulation for calculation of $\%$ drug entrapment efficacy. This determination was done in triplicate and average was calculated by using the following equation,

$\%$ entrapment efficacy $=\frac{\text { Amount of the drug loaded in nanoparticles }}{\text { Initial amount of the drug taken in formulation }} \times 100$

\section{Transmittance of Bionanosuspension}

The transmittance was measured as a function of the particle size in nano range done by sonication method. The transmittance depends on the particle size range at the particular range that defines the size particles are below range and size of the particles beyond the range required. The transmittance was determined before and after the sonication cycle. The transmittance at different wavelength indicates that when the light is passed through the particles means the particle size is below that wavelength, which indicates that $\%$ of the particles is below $400 \mathrm{~nm}$ in the mixture, and the \% blockade shows that the $\%$ of particles is above $400 \mathrm{~nm}$. The transmittance was measured by using the UV spectrophotometer. After each sonication cycle, the \% transmittance was found to be increased due to reduction of the particles to nano range. The effect of sonication on \% transmittance was observed after sonication and measuring the $\%$ transmittance after each sonication cycle. ${ }^{[18,19]}$

\section{In vitro Drug Release}

The in vitro release study was performed for all formulation. In vitro release study was performed by novel static method by using modified MS diffusion cell (Fig. 2), which consisted of one upper donor compartment and a lower receptor compartment that was used for diffusion study. The formulation for release study was 


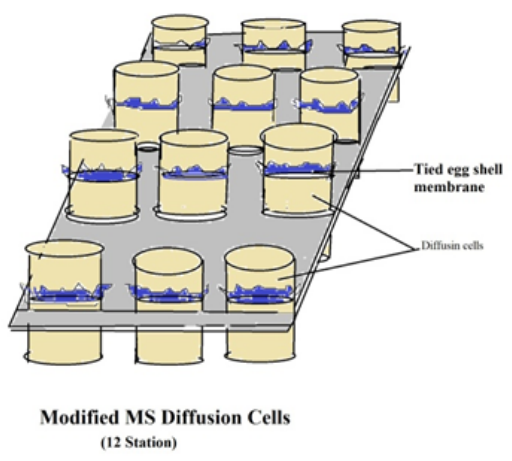

Fig. 2: Diagrammatic representation of modified Madhav-Shankar (MS) diffusion cell

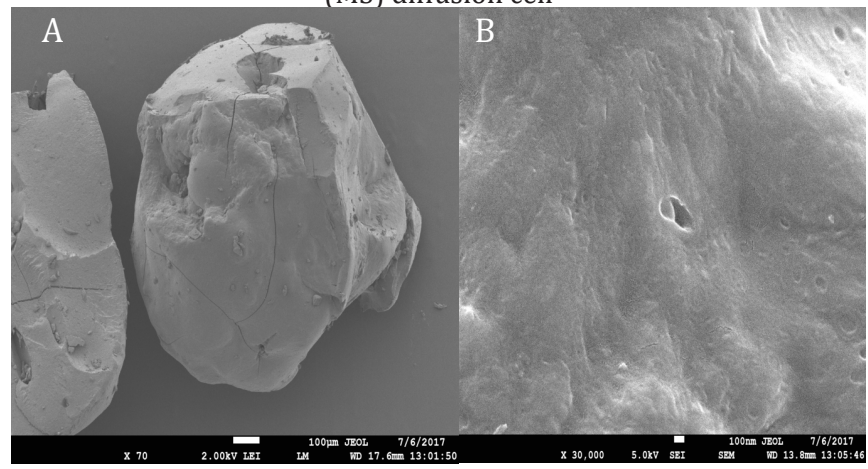

Fig. 3: SEM of isolated biopolymer of biopolymer; A: 70X; B: $30,000 \mathrm{X}$

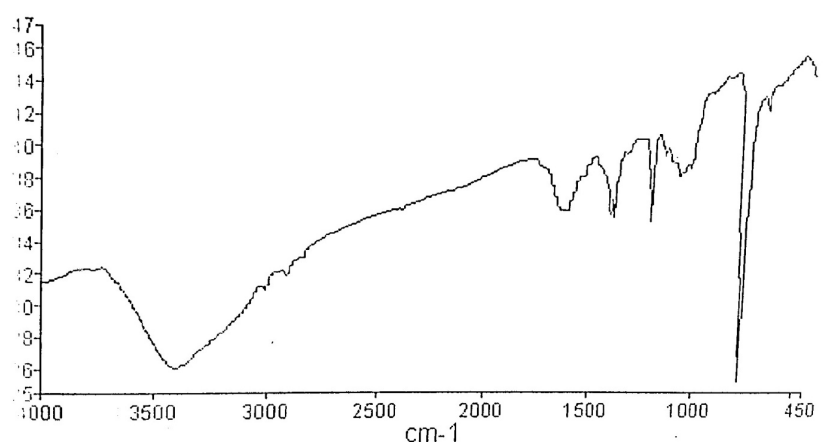

Fig. 4: FTIR spectra of biopolymer from $P$. vulgaris

taken in the donor compartment $(1 \mathrm{~mL})$, and the end of the donor is tied with the egg membrane. This donor compartment was immersed in the receiver compartment having $13 \mathrm{~mL}$ of $\mathrm{pH} 7.4$ phosphate buffer solution. Sampling was done at different regular time intervals for 36 hours. The samples were withdrawn completely and replaced with the fresh phosphate buffer solutions after every sampling. The samples were analyzed by a UV spectrophotometer for determining the released amount of the drug. The \% cumulative drug release (\% CDR) was calculated with respect to different time interval. The other parameters, like $r^{2}$ value, t $50 \%$, and $t 80 \%$, were calculated for the different formulations (LPVu1LPVu10). Apart from this, in vitro release study was also performed for nanosuspensions (LSP1-LSP10) by the same procedure.
Table 3: Physicochemical characterization of biopolymer from Phaseolus vulgaris

\begin{tabular}{ll}
\hline Parameter & Observation \\
\hline Color & Creamy-white \\
Odor/taste & Characteristic \\
Melting Point & $245 \pm 2^{\circ} \mathrm{C}$ \\
Solubility & Slightly soluble in water, \\
& soluble in acetone \\
Carbohydrate & Present \\
Protein & Present \\
\hline
\end{tabular}

Particle Size Analysis

The particle size of the bionanosuspension was studied by characterizing with the Malvern zeta sizer, ver. 6.34 .

\section{Stability}

The stability study was performed as per ICH guidelines (ICH-Q1A-second revision). The formulations were stored at different temperatures like $37 \pm 345 \pm 2$, and $60 \pm 2^{\circ} \mathrm{C}$ in hot air oven for 6 months. The samples under evaluation were observed for the drug content, $\mathrm{pH}$ changes, and also, any changes in color, odor and taste, entrapment efficacy, and in vitro release study.

\section{RESULTS AND DISCUSSION}

\section{Isolation of Biopolymer}

The $P$. vulgaris biopolymer was found to be brownishcream in color with a yield of $11 \pm 0.2 \%$.

\section{Characterization of Isolated Biopolymer}

The isolated biopolymer was creamy white in appearance. The biopolymer was found to be odorless with characteristic taste. It was found to be sparingly soluble in water. It showed positive test for carbohydrates and proteins. The characterization of isolated biopolymer of $P$. vulgaris with observation is shown in Table 3.

\section{Scanning Electron Microscopy (SEM)}

The SEM image of an isolated biopolymer of biopolymer at 30,000X and 70X magnification has shown in Fig. 1. The SEM images showed flaky structured amorphous particles. The surface of the biopolymer was smooth and amorphous nature comparable to those reported earlier. ${ }^{[20]}$

\section{IR Spectroscopy}

The IR spectra (Fig. 4) of the biopolymer shows the presence of different functional groups, like hydroxyl $\left(3,402 \mathrm{~cm}^{-1}\right)$, alkenes $\left(670 \mathrm{~cm}^{-1}\right)$, carboxylic acid $\left(1,384.99 \mathrm{~cm}^{-1}\right)$, which confirms its polymeric characteristics. The other groups like aromatic ring at $1,403.7 \mathrm{~cm}^{-1}$ due to $\mathrm{C}-\mathrm{C}$ stretching vibrations, $\mathrm{h}$ bending at $771.84 \mathrm{~cm}^{-1}$, amide at $1,610.42 \mathrm{~cm}^{-1}$, ester at $1,384 \mathrm{~cm}^{-1}$, and tertiary alcohol at $1,082 \mathrm{~cm}^{-1}$, were found to be present in the IR spectra. The presence of these functional groups is responsible 
for the retardibility in drug release like other standard polymer. ${ }^{[18]}$

\section{Differential Scanning Calorimetry (DSC)}

The DSC thermogram of the biopolymer showed endothermic peak at $73.006^{\circ} \mathrm{C}$ (Fig. 5). The area was found to be $94.3136 \mathrm{~mJ} / \mathrm{mg}$. The broad peak indicates amorphous nature of the biopolymer.

\section{NMR Spectroscopy}

The NMR spectra show the presence of different peaks at $0.011,1.559$, and 7.262 ppm (Fig. 6), which confirms the presence of methyl group, hydroxyl group, aryl proton, respectively. The ${ }^{1} \mathrm{H}$ NMR spectra confirm the presence of carbohydrate residue within the biopolymer extracted as the shift of carbohydrate ring proton from 2 to $7 \mathrm{ppm}^{[19]}$ The presence of these groups confirms its polymeric nature.

\section{Transmittance of Nanosized Lamotrigine}

During the nanosizing of lamotrigine after each sonication cycle, the sample was observed for transmittance that confirms that as the number of cycles increases, the $\%$ transmittance was increased. This was due to decrease in

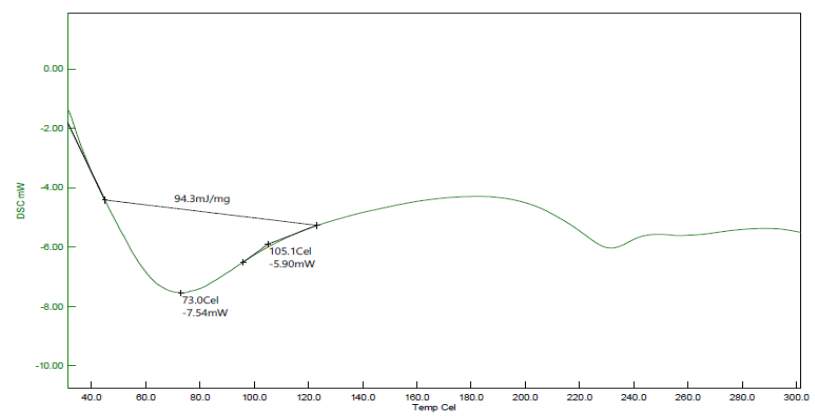

Fig. 5: Differential scanning calorimetry (DSC) of biopolymer from P. vulgaris
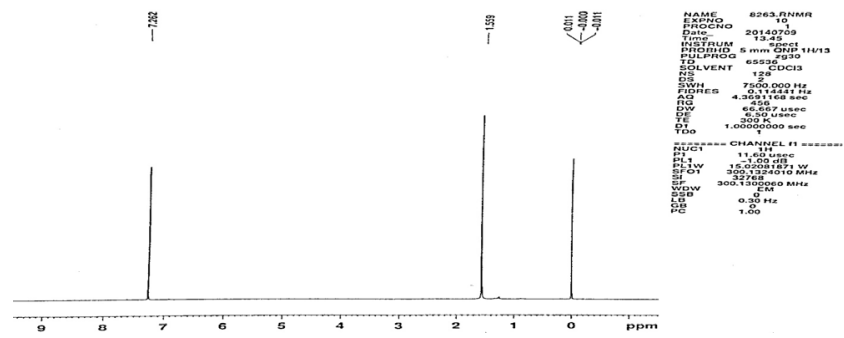

Fig. 6: NMR spectra of biopolymer from P. vulgaris

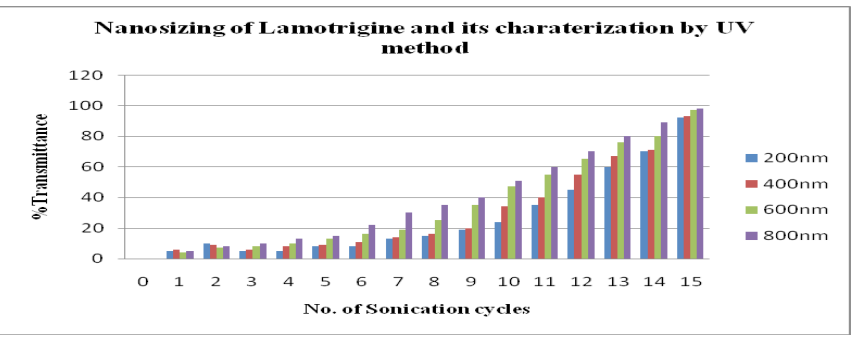

Fig. 7: Nanosizing of lamotrigine and its characterization by transmittance method particle size and particles are now are in nano range. Thus, $\%$ transmittance shows the \% of particles below $400 \mathrm{~nm}$ in bionanosuspension, and \% blockade gives an idea about the $\%$ of particles which are above $400 \mathrm{~nm}$. Thus, the UV method has given an idea about particles in nano range. ${ }^{[15]}$ The nanosizing of lamotrigine and its characterization by transmittance method is depicted in Fig. 7.

\section{Permeability through Egg Membrane}

Agraph was plotted between the drug permeated at different time intervals through the eggshell membrane (Fig. 8). The egg membrane was used as the permeation membrane, which mimics the physiology of biomembrane. ${ }^{[19]}$ Cumulative graph showed an increase in permeation with time.

\section{Drug-Excipient Interaction}

There was no change in $\lambda_{\max }$ before ( $307 \mathrm{~nm}$ ) and after the test ( $307 \mathrm{~nm}$ ) in drug excipient study. The $\lambda_{\max }$ was found to be the same as the drug-biopolymer mixture as that of pure drug. This confirms no interaction between drug and biopolymer and the isolated biopolymer can be used for the preparation of bionanosuspension.

\section{Lamotrigine-loaded Bionanosuspension}

The different formulations of bionanoparticles by using different ratios of biopolymer and lamotrigine were prepared. The nanoparticles by using different ratios of lamotrigine and hydroxypropyl methylcellulose were also prepared. The formulations were evaluated for different parameters, and their findings are described below.

\section{Dispersibility}

The dispersibility of the formulated bionanoparticles was found to be excellent. The redispersion was also found to be good. All nanoparticles remained in dispersed state during the test period. No aggregation or lump formation was observed.

$p H$

The $\mathrm{pH}$ of the bionanosuspension was found to be in the range of $\mathrm{pH} 7.1$ to 7.6 close to physiological $\mathrm{pH}$. The $\mathrm{pH}$ of different bionanosuspensions is given in Table 4.

\section{Entrapment Efficacy}

The entrapment efficacy of the prepared bionanosuspension was found to be in the range of $83.56 \pm 2$ to $89.89 \pm 1 \%$.

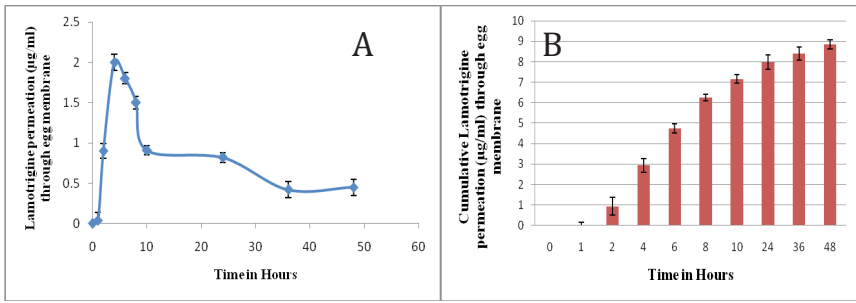

Fig. 8: Study of lamotrigine permeation through egg membrane; A. Direct concentration vs. time; B. Cumulative drug diffusion graph 
Bionanoparticles loaded with Nanosized Lamotrigine

Table 4: Different formulations with observed $\mathrm{pH}$, dispersibility, and entrapment efficacy for LPVu1-LPVu10 bionanosuspension

\begin{tabular}{llll}
\hline Formulations & Observed $p H$ & Dispersibility & $\begin{array}{l}\text { Entrapment } \\
\text { efficacy (\%) }\end{array}$ \\
\hline LPVu1 & $7.1 \pm 0.6$ & - & $83.9 \pm 2$ \\
LPVu2 & $7.4 \pm 0.36$ & - & $83.56 \pm 1.1$ \\
LPVu3 & $7.4 \pm 0.42$ & + & $84.12 \pm 1.5$ \\
LPVu4 & $7.2 \pm 0.28$ & + & $85.45 \pm 2$ \\
LPVu5 & $7.6 \pm 0.18$ & + & $84.89 \pm 3$ \\
LPVu6 & $7.4 \pm 0.41$ & + & $87.12 \pm 2$ \\
LPVu7 & $7.4 \pm 0.21$ & + & $89.89 \pm 1$ \\
LPVu8 & $7.2 \pm 0.16$ & + & $87.11 \pm 3$ \\
LPVu9 & $7.4 \pm 0.34$ & + & $88.32 \pm 4$ \\
LPVu10 & $7.5 \pm 0.23$ & - & $88.1 \pm 3$ \\
\hline
\end{tabular}

Abbreviation: + = good; - = not suitable

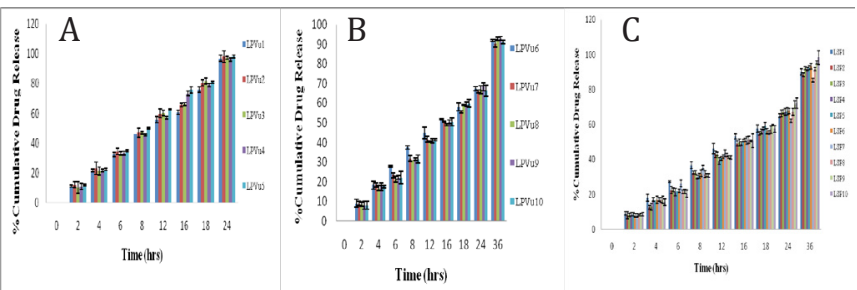

Fig. 9: In vitro drug release profile of different $P$. vulgaris bionanosuspension; A. For LPVu1-LPVu5; B. For LPVu6-LPVu10; C. HPMC nanosuspension (LSP1-LSP10)

The obtained entrapment efficacy for different formulation by using a biopolymer and HPMC has been given in Tables 4 and 5, respectively.

\section{In vitro Drug Release}

The in vitro drug release study was done by using the MS diffusion apparatus. The drug release kinetics was evaluated using the BIT-1.12 software, and t 50\%, t $80 \%$, and $\mathrm{r}^{2}$ were calculated. All the formulations showed more than $90.36 \%$ drug release (Figs $9 \mathrm{~A}$ and $\mathrm{B}$ ). The release kinetics was studied, and the formulation LPVu7 was found to be the best formulation having t $50 \%$ of 17 hours and $t 80 \%$ of 29 hours with $r^{2}$ value of 0.9927 . The best formulation LPVu7 showed up to $90.36 \pm 0.9 \%$ drug release over 36 hours. The best fit model was found to be Korsmeyer Peppas, with the mechanism of drug release to be anomalous transport. The release kinetic of all formulations indicates the sustained release of the lamotrigine from the bionanosuspension. Lamotrigine release from the bionanosuspension prepared was compared with lamotrigine loaded nanosuspension (LSP1-LSP10), using standard polymer HPMC (Fig. 9C). In case of nanosuspension prepared by using HPMC the release was found up to $92.43 \pm 0.68 \%$ with t $50 \%$ of 16 hours and $t 80 \%$ of 28 hours with $r^{2}$ value of 0.9951 over 36 hours from the best stable, optimized formulation (LSP10) with the best fit model Korsmeyer Peppas and
Table 5: Nanoformulations made with hydroxypropyl methylcellulose (HPMC): $\mathrm{pH}$, dispersibility, and entrapment efficacy for LSP1-LSP10

\begin{tabular}{llll}
\hline Formulations & Observed $p H$ & Dispersibility & $\begin{array}{l}\text { Entrapment } \\
\text { efficacy (\%) }\end{array}$ \\
\hline LSP1 & $7.2 \pm 0.2$ & - & $76.9 \pm 2$ \\
LSP2 & $7.3 \pm 0.3$ & - & $75.56 \pm 1.1$ \\
LSP3 & $7.4 \pm 0.5$ & + & $79.12 \pm 1.5$ \\
LSP4 & $7.4 \pm 0.3$ & + & $80.45 \pm 2$ \\
LSP5 & $7.4 \pm 0.1$ & + & $84.89 \pm 3$ \\
LSP6 & $7.4 \pm 0.3$ & + & $84.12 \pm 2$ \\
LSP7 & $7.3 \pm 0.4$ & + & $81.89 \pm 1$ \\
LSP8 & $7.5 \pm 0.2$ & - & $85.11 \pm 3$ \\
LSP9 & $7.6 \pm 0.2$ & - & $69.32 \pm 4$ \\
LSP10 & $7.5 \pm 0.23$ & - & $73.1 \pm 3$ \\
\hline
\end{tabular}

Abbreviation: + = good; - = not suitable
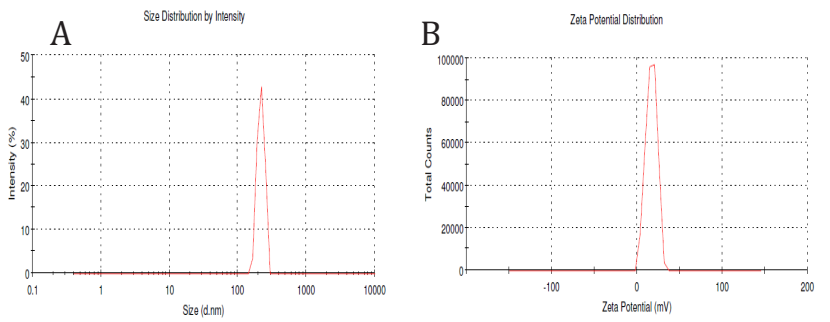

Fig. 10: Particle size distribution and zeta potential distribution in bionanosuspension

anomalous transport as the mechanism of drug release in kinetic study. The best formulation LPVu7 was subjected to particle size analysis.

\section{Particle Size}

The average particle size of LPVu7 bionanosuspension was found to be $661.2 \mathrm{~nm}$ with a zeta potential of $16.7 \mathrm{mV}$ confirms that the nanoparticles are in nano range, which is responsible for the stability of nanosuspension. The result reveals that it can be safely used for the delivery of lamotrigine in treatment of epilepsy. The particle size distribution in bionanosuspension and zeta potential distribution of bionanosuspension are shown in Fig. 10.

\section{Stability}

The optimized formulation showed no change in $\lambda_{\max }$ entrapment efficacy, and \%cumulative drug release, so there was no drug loss during the study period. There was no change in color, odor, $\mathrm{pH}$, and physical appearance. The evaluated parameters confirmed that the formulation was physically and chemically stable.

\section{Conclusion}

The research concludes that the isolated biopolymer from the seeds of $P$. vulgaris consists of novel inbuilt stabilizer cum retardant properties, which can be effectively used for the development of lamotrigine loaded bionanosuspension for delivery of lamotrigine in a sustained manner. 
Sushant Kumar et al.

\section{ACKNOWLEDGMENT}

The authors wish to acknowledge Prof. Devender Pathak (Dean, Faculty of Pharmacy, UPUMS, Saifai), Ms. Swarnima Pandey (Assistant Professor, AKTU, Lucknow) for encouraging them for the completion of research work. The authors also want to thank SAIF, CDRI, Lucknow for providing them with the analytical testing facilities.

\section{REFERENCES}

1. Satheesh Madhav NV, Tangri P. Formulation and evaluation of zidovudine bio-micro dwarfs using a novel bio muco resident from Artocarpus heterophyllus. Int J. Pharm Tech Res. 2011;3:169-174.

2. Satheesh Madhav NV, Shankar MSU. A novel smart mucoadhesive biomaterial from Lallimantia royalena seed coat. J Sci Asia. 2011;37:69-71.

3. Satheesh Madhav NV, Pangatey P. Indian Patent: Development of flexy bio- adhesive film loaded with nanosized zidovudine for brain specificity via novelistic soft palatal route, 2015.

4. Satheesh Madhav NV, Tangri P. Design and evaluation of insulin loaded Bio-Flexy films for trans-soft palatal to brain delivery. Int J Therap Applicat. 2012;4:10-18.

5. Satheesh MNV, Yadav AP. Development and evaluation of novel repaglinide biostrips for translabial delivery. Int. Res. J. Pharm. 2013;4:198-202.

6. Ojha A, Satheesh MNV. A novel potent muco-bioadhesant polymer from seeds of Ricinus communis. World J. Pharm. Pharmaceut. Sci. 2014;3:2154-2165.

7. Satheesh MNV, Yadav AP. A novel translabial platform utilizing bioexcipients from Litchi chinensis for the delivery of rosiglitazone maleate. Acta Pharm Sinica. B. 2013;3,408-415.

8. Muller. Nanosuspensions as particulate drug formulation in therapy rationale for development and what we can expect for the future.
Adv Drug Deliv Rev. 2001;47:3-19.

9. Rogawski MA, Loscher W. The neurobiology of antiepileptic drugs. Nat. Rev. Neurosci. 2004;5:553-564.

10. Zhang J. Preparation of amorphous cefuroxime axetil nanoparticles by controlled nanoprecipitation method without surfactants. Int J Pharm. 2006;323:153-163.

11. Patsalos PN, Froscher W, Pisani F. The importance of drug interactions in epilepsy therapy. Epilepsia. 2002;43:365-385.

12. Dawood NM. Formulation and characterization of lafutidine nanosuspension for oral drug delivery system. Int J Appl Pharm. 2018;10:20-30.

13. Madhav S, Singh K. Smart ungual biopenetrant from the roots of Beta vulgaris. Journal of Appl Pharm Res. 2017;5(2):21-26.

14. Martin A. Physical, Chemical principles in the pharmaceutical sciences. 3rd ed. Varghese Publishing House, Bombay, 2001.

15. Varshney S, Satheesh MNV. Smart approach for preparing nanosized topiramate loaded bio-flexy films using former from piper nigrum and screening its in vitro performance. Nanomedicine and Nanotechnology. 2018;3(3):000147.

16. Subrahmanyam CVS, Thimma Setty.J. Laboratory Manual of Physical Pharmaceutics. 2nd ed. Vallabh Prakashan, New Delhi, 2002.

17. Tyagi Y, Satheesh MNV. Design selegiline loaded bio-nanosuspension for the management of depression using novel bio-retardent from Manilkara zapota. Drug Development and in Industrial Pharmacy. 2019;45:1351-1360.

18. Kamila SN, Satheesh MNV, Sarkar CN. Evaluation of effective Medhya formulation on trans-cranial treatment on rat. Int J Biomed Res. 2014;5(6):427-431.

19. Satheesh MN, Singh B. A smart approach for delivering of nanosized olanzapine using piper betel biopolymer rate controlling flexi films for transvermillion delivery. Asian Journal of Nanoscience and Materials. 2019;02:314-326.

20. Satheesh MNV, Raina D. Formulation and evaluation of duloxetine loaded bio-nanosuspension for brain specificity via acoustic meatus. SOJ Pharmacy \& Pharmaceutical Science. 2017;4(1):1-5.

HOW TO CITE THIS ARTICLE: Kumar S, Madhav NVS, Verma A, Pathak K. Development and evaluation of novel bionanoparticles loaded with nanosized lamotrigine by using the novel isolated biopolymer from Phaseolus vulgaris seeds. Int. J. Pharm. Sci. Drug Res. 2020;12(3):247-254. DOI: $10.25004 /$ IJPSDR.2020.120306 